\title{
BOUNDED UNIVALENT FUNCTIONS
}

\author{
BY
}

RAPHAEL M. ROBINSON

1. Introduction. We shall consider the class of functions $f(z)$ which are regular and univalent for $|z|<1$, with $|f(z)|<1$ there, and with $f(0)=0$. For any fixed $z_{0} \neq 0$ in the unit circle, we use the abbreviations

$$
a=\left|f^{\prime}(0)\right|, \quad b=\left|z_{0}\right|, \quad c=\left|f\left(z_{0}\right)\right|, \quad d=\left|f^{\prime}\left(z_{0}\right)\right| .
$$

We are concerned in this paper with the inequalities relating $a, b, c, d$. It may be noted that these relations are not affected if we impose the condition $f^{\prime}(0)>0$, which we shall do.

The four quantities (1) are restricted individually only by

$$
0<a \leqq 1, \quad 0<b<1, \quad 0<c<1, \quad 0<d .
$$

If $a=1$, then $f(z)=z$, hence $d=1$ and $c=b$. This trivial case will be excluded below where convenient. If $a$ has any other given value, then it is easily seen that no restriction is placed on any one of the other quantities. Between $b$ and $c$ the only relation is $c \leqq b$; the equality $c=b$ holds only if $a=1$. The relations among the quantities of each of the sets $(b, d),(c, d),(a, b, c),(b, c, d)$, and $(a, b, c, d)$ are considered in $\$ 5$. The relations between $(a, b, d)$ are considered in $\S 6$, and those between $(a, c, d)$ in $\S 7$. Thus all subsets of the four quantities are considered. It should be pointed out that the determination of the inequalities satisfied by the four quantities by no means completes the solution, since one of the main difficulties is that of eliminating one of the quantities in order to find the inequalities satisfied by three of them.

All of the inequalities which we obtain will be sharp; that is, in each case there is an extremal function for which the inequality becomes an equality. But in general we shall not go beyond the mere existence of such an extremal function.

Finally, there is an appendix ( $\$ 8)$ on unbounded univalent functions. Let $F(z)$ be regular and univalent for $|z|<1$, and suppose $F(0)=0, F^{\prime}(0)=1$. The relations between $\left|z_{0}\right|,\left|F\left(z_{0}\right)\right|$, and $\left|F^{\prime}\left(z_{0}\right)\right|$ are discussed in detail. One result, which may seem surprising, will be mentioned here: If $\left|F\left(z_{0}\right)\right| \leqq 1 / 4$ then

$$
\left|F^{\prime}\left(z_{0}\right)\right| \leqq 1+3 \cdot 2^{-3 / 2}=2.06 \cdots,
$$

but if $\left|F\left(z_{0}\right)\right|$ has a prescribed value greater than $1 / 4$, no upper bound for $\left|F^{\prime}\left(z_{0}\right)\right|$ can be given. The results in the appendix are obtained as limiting cases of results for bounded univalent functions, but without using $\S 6$ and $\$ 7$.

Presented to the Society, November 22, 1941; received by the editors November 13, 1941 . 
2. The method of Löwner. If, from our class of bounded univalent functions, a subclass is chosen by means of which any function of the given class can be uniformly approximated in the interior of the unit circle, then the inequalities between any of the quantities $a, b, c, d$ are the same for the subclass as for the whole class, except perhaps as regards the possibility of equality signs holding.

According to Löwner( $\left.{ }^{1}\right)$, we may choose the subclass as the class of functions $f(z)$ to which a function $f(z, t)$ can be found, with the following properties. There is a number $I>0$ such that $f(z, t)$ is continuous for $|z|<1$ and $0 \leqq t \leqq I$, and is a regular function of $z$ for each fixed $t$. The boundary conditions

$$
f(z, 0)=z, \quad f(z, I)=f(z)
$$

are satisfied, so that $f(z)$ may be regarded as having been obtained from the identity by continuous variation. The rate at which this variation takes place is governed by

$$
f^{\prime}(0, t)=e^{-t},
$$

where the prime denotes differentiation with respect to $z$. Finally, there is a continuous function $\kappa(t)$ with $|\kappa(t)|=1$, such that $f(z, t)$ satisfies the differential equation

$$
\frac{\partial f(z, t)}{\partial t}=-f(z, t) \frac{1+\kappa(t) f(z, t)}{1-\kappa(t) f(z, t)} .
$$

It is also permissible, and more convenient for us, to allow $\kappa(t)$ to be a piece-wise continuous function; we then understand that (5) is to hold except at the points of discontinuity of $\kappa(t)$, and similarly below. This weaker condition on $\kappa(t)$ means that we are choosing a larger subclass from the class of bounded univalent functions. The advantage of this is that extremal functions for all of our inequalities are then brought within the subclass.

Another fact which is important for us is that (5) can be solved for any given $\kappa(t)$ satisfying the conditions mentioned, so that there is a one-to-one correspondence between such functions $k(t)$ and functions $f(z)$ of our subclass.

From (3) and (4) we see that

$$
a=e^{-I} \text {. }
$$

3. The integrals $I$ and $J$. From (5) we readily obtain

$$
\frac{d}{d t} \log \left|f\left(z_{0}, t\right)\right|=-\frac{1-\left|f\left(z_{0}, t\right)\right|^{2}}{\left|1-\kappa(t) f\left(z_{0}, t\right)\right|^{2}} .
$$

(1) K. Löwner, Untersuchungen über schlichte konforme Abbildungen des Einheitskreises, Mathematische Annalen, vol. 89 (1923), pp. 103-121. 
If we put

$$
s=\left|f\left(z_{0}, t\right)\right|,
$$

then $s$ decreases from $b$ to $c$ while $t$ increases from 0 to $I$. Any function of $t$ in the interval $0 \leqq t \leqq I$ may be regarded as a function of $s$ in the interval $c \leqq s \leqq b$. In particular, we put

$$
\kappa(t) f\left(z_{0}, t\right)=\eta(s) s,
$$

so that $\eta(s)$ is a piece-wise continuous function with $|\eta(s)|=1$. Then (7) takes the form

$$
\frac{d s}{d t}=-\frac{s}{H(s)}
$$

where

$$
H(s)=\frac{|1-\eta(s) s|^{2}}{1-s^{2}}
$$

We have evidently

$$
\frac{1-s}{1+s} \leqq H(s) \leqq \frac{1+s}{1-s} .
$$

We show now that $\eta(s)$ is an arbitrary piece-wise continuous function with $|\eta(s)|=1$. If any such $\eta(s)$ is given in the interval $c \leqq s \leqq b$, then we first determine $H(s)$ from (11), and then from (10) and the fact that $t=0$ for $s=b$, we find that

$$
t=\int_{0}^{b} H(\xi) d \xi / \xi
$$

and in particular that

$$
I=\int_{c}^{b} H(s) d s / s
$$

Now (13) determines $s$ as a function of $t$ in the interval $0 \leqq t \leqq I$, so that (8) and (9) determine $\left|f\left(z_{0}, t\right)\right|$ and $\kappa(t) f\left(z_{0}, t\right)$. From (5) it follows that

$$
\frac{d}{d t} \operatorname{amp} f\left(z_{0}, t\right)=-\frac{2 \Im\left[\kappa(t) f\left(z_{0}, t\right)\right]}{\left|1-\kappa(t) f\left(z_{0}, t\right)\right|^{2}} .
$$

Using the value just found for $\kappa(t) f\left(z_{0}, t\right)$, we can find $\operatorname{amp} f\left(z_{0}, t\right)$, and hence $f\left(z_{0}, t\right)$ itself, the initial condition $f\left(z_{0}, 0\right)=z_{0}$ being imposed. Finally, knowing $\kappa(t) f\left(z_{0}, t\right)$ and $f\left(z_{0}, t\right)$, we can find $\kappa(t)$ by division; it will be piece-wise continuous and satisfy $|\kappa(t)|=1$. Now the $\kappa(t)$ and $f\left(z_{0}, t\right)$ determined satisfy (7) 
and (15), which are equivalent to (5) with $z=z_{0}$. From this we see that the $\kappa(t)$ which we have found will in fact lead back to the desired $\eta(s)$.

From the fact that $\eta(s)$ is arbitrary, we see that $H(s)$ is an arbitrary piecewise continuous function satisfying (12). For if any such $H(s)$ is given, we can find an $\eta(s)$ satisfying (11).

It is readily seen that if $f(z, t)$ is a continuous function which is regular in $z$ for each fixed $t$, and such that $f_{t}(z, t)$ is continuous, then $f_{z t}(z, t)$ and $f_{t z}(z, t)$ exist and are equal. Using this fact, from (5) we find that

$$
\frac{d}{d t} \log \left|f^{\prime}\left(z_{0}, t\right)\right|=1-2 \frac{\Re\left\{\left[1-\kappa(t) f\left(z_{0}, t\right)\right]^{2}\right\}}{\left|1-\kappa(t) f\left(z_{0}, t\right)\right|^{4}} .
$$

Expressing the right side in terms of $s$, and using (10), we have

$$
\frac{d}{d t} \log \left|f^{\prime}\left(z_{0}, t\right)\right|=\frac{-1}{s\left(1-s^{2}\right)}\left[|1-\eta(s) s|^{2}-2 \frac{\Re\left\{[1-\eta(s) s]^{2}\right\}}{|1-\eta(s) s|^{2}}\right] \frac{d s}{d t} .
$$

Integrating from $t=0$ to $t=I$ gives

$$
\log d=\int_{c}^{b} \frac{|1-\eta(s) s|^{2}-2 \cos \{2 \operatorname{amp}[1-\eta(s) s]\}}{s\left(1-s^{2}\right)} d s .
$$

Now

$$
\cos \operatorname{amp}[1-\eta(s) s]=\frac{1-s^{2}+|1-\eta(s) s|^{2}}{2|1-\eta(s) s|},
$$

so that the numerator of the integrand becomes $2 s^{2}-\left(1-s^{2}\right) / H(s)$, and (17) takes the form

$$
d=\frac{1-c^{2}}{1-b^{2}} e^{-J}
$$

where

$$
J=\int_{c}^{b}(1 / H(s)) d s / s .
$$

The problem of finding what values are possible for $a$ and $d$ when $b$ and $c$ are given is thus reduced to finding the relations between the integrals $I$ and $J$. The corresponding values of $a$ and $d$ are then found from (6) and (18).

4. Relations between $I$ and $J$. Suppose $0<c<b<1$, and let $I$ and $J$ be defined by (14) and (19), where $H(s)$ is any function satisfying (12) and piecewise continuous in the interval $c \leqq s \leqq b$. In this section, we shall determine the inequalities relating $I$ and $J$. It is clear that the relation between $I$ and $J$ is a symmetric one.

We start by introducing two functions which we shall need in this discussion. For $c \leqq r \leqq b$, let 


$$
\begin{aligned}
& p(r ; b, c)=\int_{c}^{r} \frac{1-s}{1+s} \frac{d s}{s}+\int_{r}^{b} \frac{1-r}{1+r} \frac{d s}{s}, \\
& q(r ; b, c)=\int_{c}^{r} \frac{1+s}{1-s} \frac{d s}{s}+\int_{r}^{b} \frac{1+r}{1-r} \frac{d s}{s} .
\end{aligned}
$$

Evaluating the integrals gives

$$
\begin{aligned}
& p(r ; b, c)=\log \left[\frac{r}{(1+r)^{2}}: \frac{c}{(1+c)^{2}}\right]+\frac{1-r}{1+r} \log \frac{b}{r} \\
& q(r ; b, c)=\log \left[\frac{r}{(1-r)^{2}}: \frac{c}{(1-c)^{2}}\right]+\frac{1+r}{1-r} \log \frac{b}{r}
\end{aligned}
$$

in particular we have

$$
\begin{array}{ll}
p(b ; b, c)=\log \left[\frac{b}{(1+b)^{2}}: \frac{c}{(1+c)^{2}}\right], & p(c ; b, c)=\frac{1-c}{1+c} \log \frac{b}{c}, \\
q(b ; b, c)=\log \left[\frac{b}{(1-b)^{2}}: \frac{c}{(1-c)^{2}}\right], & q(c ; b, c)=\frac{1+c}{1-c} \log \frac{b}{c} .
\end{array}
$$

It is clear from (20) that $p(r ; b, c)$ is a decreasing function of $r$, and $q(r ; b, c)$ an increasing function. Since $p(r ; b, c)<q(r ; b, c)$, we have in particular

$$
p(b ; b, c)<p(c ; b, c)<q(c ; b, c)<q(b ; b, c) .
$$

It is clear first of all that individually $I$ and $J$ are restricted only by the conditions

$$
\begin{aligned}
& p(b ; b, c) \leqq I \leqq q(b ; b, c), \\
& p(b ; b, c) \leqq J \leqq q(b ; b, c) .
\end{aligned}
$$

To find the largest possible value of $J$ when $I$ is given, we note that $H(s)+1 / H(s) \leqq(1-s) /(1+s)+(1+s) /(1-s)$, and hence

$$
I+J \leqq p(b ; b, c)+q(b ; b, c) .
$$

The equality is attained for any piece-wise continuous function $H(s)$ which is equal to $(1-s) /(1+s)$ in some subintervals, and to $(1+s) /(1-s)$ in others. Since these are the values of $H(s)$ which give $I$ its smallest and largest values, we see that any possible value of $I$ can be obtained in this way. Hence for any given $I$, the largest possible $J$ is determined from (26).

It remains to find the smallest possible $J$ for a given $I$. Let $k$ be any positive constant, and consider

$$
I+k^{2} J=\int_{c}^{b}\left[H(s)+\frac{k^{2}}{H(s)}\right] \frac{d s}{s} .
$$


The integrand is smallest when $H(s)=k$; but this may not be compatible with (12). It is clear that the integral will be minimized if we keep $H(s)$ as near to $k$ as possible. If $k$ is sufficiently small, then this $H(s)$ is always equal to $(1-s) /(1+s)$; and if $k$ is sufficiently large, then $H(s)=(1+s) /(1-s)$. Hence for a suitable value of $k, I$ may be given any possible value. Thus the minimum $J$ for any given $I$ is obtained for $H(s)$ as near to some constant $k$ as possible.

To formulate the lower bound for $J$, we distinguish three cases:

$$
\begin{array}{ll}
\text { Case } p . & p(b ; b, c) \leqq I \leqq p(c ; b, c) . \\
\text { Case } o . & p(c ; b, c) \leqq I \leqq q(c ; b, c) . \\
\text { Case } q . & q(c ; b, c) \leqq I \leqq q(b ; b, c) .
\end{array}
$$

We note first that $p(r ; b, c)$ is given by (14) where $H(s)$ is as near to $(1-r) /(1+r)$ as possible. Hence in Case $p$, we determine $r$ so that $p(r ; b, c)=I$, and then $J \geqq q(r ; b, c)$. Similarly, in Case $q, J \geqq p(r ; b, c)$, where $q(r ; b, c)=I$. In Case $o, H(s)$ may be equal to a constant $k$ throughout the interval, and hence $J$ is minimized in this way. Hence for $I=k \log b / c$, the minimum $J$ is $(1 / k) \log b / c$, so that $J \geqq(\log b / c)^{2} / I$. The three cases may be combined in the form

$$
J \geqq L(I ; b, c)
$$

where

$$
L(I ; b, c)=\left\{\begin{array}{lr}
q(r ; b, c), \text { where } p(r ; b, c)=I, & \text { in Case } p, \\
(\log b / c)^{2} / I, & \text { in Case } o, \\
p(r ; b, c), \text { where } q(r ; b, c)=I, & \text { in Case } q .
\end{array}\right.
$$

5. The simpler cases of the problem. In this section we obtain the inequalities among each set of quantities chosen from $(a, b, c, d)$, except for the trivial cases treated in the introduction, and the cases $(a, b, d)$ and $(a, c, d)$, which have separate sections devoted to them. However, partial results for those two cases are given here.

Relations between $a, b, c$. These were first obtained by Pick $\left({ }^{2}\right)$; more recently, Golusin $\left({ }^{3}\right)$ derived them, using the method of Löwner. From (24) we have, for $c<b$,

$$
\frac{c}{(1-c)^{2}}: \frac{b}{(1-b)^{2}} \leqq a \leqq \frac{c}{(1+c)^{2}}: \frac{b}{(1+b)^{2}} .
$$

(2) G. Pick, Über die konforme Abbildung eines Kreises auf ein schlichtes und zugleich beschränktes Gebiet, Sitzungsberichte Akademie der Wissenschaften, Vienna, vol. 126 (1917), pp. 247-263.

(3) G. M. Golusin, Über die Verzerrungssätze der schlichten konformen Abbildungen (Russian with German summary), Matematicheskii Sbornik (Recueil Mathématique), vol. 43 (1936), pp. 127-135. 
For $c=b$, (31) gives $a=1$, which is correct. Thus for any given $b$ and $c$, with $c \leqq b$, the value of $a$ is restricted only by (31). It may be noted that (31) itself implies that $c \leqq b$, so that it is in fact the only relation between $a, b, c$. by

The bounds in (31) are attained for the function $w=f(z)$ defined for $|z|<1$

$$
\frac{w}{(1-w)^{2}}=\frac{a z}{(1-z)^{2}}
$$

This function maps $|z|<1$ on $|w|<1$ with a slit along the negative real axis. The lower and upper bounds are attained for $z_{0}>0$ and $z_{0}<0$, respectively.

Relations between $b, c, d$. From (25) we have

$$
\frac{c}{(1-c)^{2}}: \frac{b}{(1-b)^{2}} \leqq d \frac{1-b^{2}}{1-c^{2}} \leqq \frac{c}{(1+c)^{2}}: \frac{b}{(1+b)^{2}} .
$$

This inequality could also be obtained from (31) by making linear transformations of $|z|<1$ and $|w|<1$ into themselves, in such a way that 0 and $z_{0}$ are interchanged in the $z$-plane, and 0 and $f\left(z_{0}\right)$ in the w-plane. From (33) we find that

$$
\frac{c(1+c)}{1-c}: \frac{b(1+b)}{1-b} \leqq d \leqq \frac{c(1-c)}{1+c}: \frac{b(1-b)}{1+b} .
$$

This is the only relation between $b, c, d$. The bounds are attained for the function (32), for $z_{0}<0$ and $z_{0}>0$, respectively.

Relations between $b$ and $d$. This case may be solved by seeing what bounds are given for $d$ by (34) when $b$ is given but $c$ is not. The only restriction on $c$ is that $0<c \leqq b$. Letting $c \rightarrow 0$, we see that there is no positive lower bound for $d$. On the other hand, the right side of (34) is bounded, and in fact has its largest value for $c=2^{1 / 2}-1$; if this is not within the allowed interval, then the largest possible value is at $c=b$. From this we find that

$$
d \leqq \begin{cases}1 & \text { if } 0<b \leqq 2^{1 / 2}-1 \\ \left(3-2^{3 / 2}\right) \frac{1+b}{b(1-b)} & \text { if } \quad 2^{1 / 2}-1 \leqq b<1 .\end{cases}
$$

This is the only relation between $b$ and $d$. The equality sign holds for the identity in the first case, and for the function (32) with $a$ chosen so that $c=2^{1 / 2}-1$ in the second. Dieudonné( $\left.{ }^{4}\right)$ has shown that the first part of (35) holds for bounded functions which are not supposed univalent.

Relations between $c$ and $d$. Letting $b \rightarrow 1$ in (34), we see that there is no restriction on the value of $d$ if only $c$ is given.

(4) J. Dieudonné, Recherches sur quelques problèmes relatifs aux polynômes et aux fonctions bornées d'une variable complexe, Annales de l'École Normale, vol. (3) 48 (1931), p. 352. 
Relations between $a, b, d$ (partial results). From (31) and (34) it is possible to find the smallest value of $d$ for given $a$ and $b$, and in some cases also the largest $d$.

If $a$ and $b$ are given, we may determine the smallest possible value of $c$ from the right side of (31), and then the smallest possible $d$ for this $c$ and the given $b$ from the left side of (34). Since the two functions of $c$ involved in the bounds are increasing, and since the equality signs in the two cases are attained together, we obtain in this way the best lower bound for $d$ in terms of $a$ and $b$ :

$$
d \geqq \frac{c(1+c)}{1-c}: \frac{b(1+b)}{1-b},
$$

where $c$ is determined from

$$
\frac{c}{(1+c)^{2}}=\frac{a b}{(1+b)^{2}} .
$$

The equality in (36) is attained for (32) with $z_{0}<0$.

Similarly, the equality signs on the left side of (31) and the right side of (34) are attained together. But the function $c(1-c) /(1+c)$, which occurs on the right side of (34), is increasing only for $c \leqq 2^{1 / 2}-1$, so that we can draw the conclusion

$$
d \leqq \frac{c(1-c)}{1+c}: \frac{b(1-b)}{1+b},
$$

where $c$ is determined from

$$
\frac{c}{(1-c)^{2}}=\frac{a b}{(1-b)^{2}},
$$

only if that value of $c$ is not greater than $2^{1 / 2}-1$. In this case, the equality will be attained for (32) with $z_{0}>0$. It is clear that we obtain in this way the best upper bound for $d$ in terms of $a$ and $b$ if $b \leqq 2^{1 / 2}-1$. We shall show in $\S 6$ that the same is true whenever $b \leqq 1 / 2$ and in some other cases, but not in all cases.

Relations between $a, c, d$ (partial results). We try to find bounds for $d$ in terms of $a$ and $c$ from (31) and (34). If

$$
a>\frac{4 c}{(1+c)^{2}},
$$

then the right side of (31) determines a largest value possible for $b$, and then with this $b$ and the given $c$, a lower bound for $d$ is determined from the left side of (34). This bound is the best possible. It is given by (36) with $b$ determined from (37), and is attained for (32) with $z_{0}<<0$. On the other hand, if 
(40) is not satisfied, then $b$ is permitted values arbitrarily close to 1 . It is easily shown that no positive lower bound exists for $d$ in this case. For example, it is sufficient to consider the functions $w=f(z)$ which map $|z|<1$ on $|w|<1$ with a slit from -1 nearly to $-c$ and a slit on the positive real axis long enough to give $a$ the proper value, $z_{0}$ being chosen so that $f\left(z_{0}\right)=-c$.

We can draw the conclusion that an upper bound for $d$ is given by (38) with $b$ determined from (39) only if $b(1-b) /(1+b)$ has its smallest value when $b$ has its smallest possible value; if this is true, then the maximum value of $d$ is attained for (32) with $z_{0}>0$. The condition is certainly satisfied if for the given values of $a$ and $c$ one has necessarily $b \leqq 2^{1 / 2}-1$; this is true at least for $a$ near 1 and $c$ near 0 . More generally, if we denote the smallest possible value of $b$ by $b_{\min }$, and suppose that $b$ has a largest possible value $b_{\max }$, then the condition is satisfied if $b(1-b) /(1+b)$ is not larger at $b_{\min }$ than at $b_{\max }$. This condition reduces to

$$
\left(1+b_{\min }\right)\left(1+b_{\max }\right) \leqq 2,
$$

which is the best result obtainable by the present method. We shall show in $\S 7$ that the conclusion holds if and only if

$$
b_{\min } \leqq 1 / 2, \quad b_{\max } \leqq 1,
$$

where the second inequality is to be interpreted to mean: either $a$ and $c$ have such values that $b_{\max }<1$, or are limits of such values.

Remark on the hyperbolic expansion factor. We may interpret the expression $d\left(1-b^{2}\right) /\left(1-c^{2}\right)$, which occurs in (33), as the expansion factor for the mapping $w=f(z)$, when the metric of hyperbolic geometry is introduced in $|z|<1$ and $|w|<1$. By a similar argument to that used above, we find that no matter which two of the three quantities $a, b, c$ are given, the hyperbolic expansion is minimized for (32) with $z_{0}<0$, and maximized for (32) with $z_{0}>0$. Only in case $a$ and $c$ are given, not satisfying (40), and we are seeking to minimize the hyperbolic expansion, is it impossible to satisfy the necessary conditions, $z_{0}<0$ and $\left|f\left(z_{0}\right)\right|=c$. But in this case we know that $d$ has no positive lower bound, and a fortiori the same is true of the hyperbolic expansion. It may also be noted that the conclusion that the hyperbolic expansion has its extreme values for (32) is weaker than the same conclusion about $d$, and hence follows from this when this is true. The bounds for the hyperbolic expansion when $a$ and $b$ are given were found by Pick $\left(^{5}\right)$.

So far in this section, we have used from $\$ 4$ only the trivial results (24) and (25); the rest of the section is used first in considering the relation between all four quantities. It may also be noted that the results of this section so far have depended only on (31) and (33). Since (33) can be deduced from (31), these results can be obtained without using Löwner's method, if we as-

(5) Pick, loc. cit. 
sume (31) from the work of Pick. The rest of this section, and the next two sections, depend essentially on Löwner's method.

Relations between $a, b, c, d$. On account of (18), we see that the lower bound for $d$ in terms of $a, b, c$ is found from the upper bound for $J$, given in (26). This leads to

$$
d \geqq \frac{1}{a} \frac{c^{2}}{1-c^{2}}: \frac{b^{2}}{1-b^{2}} .
$$

It may be verified that this bound is attained for any function $w=f(z)$ mapping $|z|<1$ on $|w| \cdot<1$ with slits along the positive and negative real axes; the equality sign in (42) then holds for any positive or negative $z_{0}$. The lengths of the slits and the value of $z_{0}$ may be so chosen as to give any desired values to $a, b, c$.

Similarly, the upper bound for $d$ is found from the lower bound for $J$, given by (29). The result may be written in the form

$$
\log d \leqq M(I ; b, c),
$$

where

$$
M(I ; b, c)=\log \frac{1-c^{2}}{1-b^{2}}-L(I ; b, c) .
$$

6. Relations between $a, b, d$. We shall obtain the lower and upper bounds for $d$ in terms of $a$ and $b$ by eliminating $c$ from (42) and from (43); this will complete the partial solution given in $\$ 5$.

The lower bound for $d$ in terms of $a$ and $b$ may be obtained from (42) by substituting the smallest possible value of $c$, which is obtained from (37). This is seen to agree with our previous result, which was (36) with the same value of $c$ substituted.

We turn now to the problem of finding the upper bound for $d$ in terms of $a$ and $b$. We have to maximize $M(I ; b, c)$ for all possible values of $c$. Now (44) defines $M(I ; b, c)$ in terms of $L(I ; b, c)$, which in turn is defined by (30). In (30), different formulas hold in each of the three cases (28). The cases are distinguished according to the interval in which $I$ lies when $b$ and $c$ are given. But now we wish to consider $I$ and $b$ as given, and see in what intervals $c$ must lie in order that each of the cases may hold.

We note first that all four functions $p(b ; b, c), p(c ; b, c), q(c ; b, c), q(b ; b, c)$ are decreasing functions of $c$. This is evident from (22) for all the functions but $q(c ; b, c)$. For fixed $b$, let $\phi(c)=q(c ; b, c)$; then

$$
\phi(c)=\frac{1+c}{1-c} \log \frac{b}{c}, \quad \phi^{\prime}(c)=\frac{2}{(1-c)^{2}} \log \frac{b}{c}-\frac{1+c}{c(1-c)} .
$$

If we put $\psi(c)=\log c+\left(1-c^{2}\right) / 2 c$, then the condition $\phi^{\prime}(c)<0$ reduces to the 
form $\psi(c)>\log b$. Now $\psi^{\prime}(c)=-(1-c)^{2} / 2 c^{2}:<0$, so that $\psi(c)$ is decreasing. Hence $\psi(c)>\psi(b)>\log b$, as was to be shown.

Thus each of the four functions is strictly monotone, and from (22) it is seen that each decreases from $+\infty$ to 0 as $c$ increases from 0 to $b$. Hence if $a$ and $b$ are given, with $a<1$, there are unique numbers $c_{p}, \bar{c}_{p}, \bar{c}_{q}, c_{q}$, between 0 and $b$, which satisfy

$$
p\left(b ; b, c_{p}\right)=p\left(\bar{c}_{p} ; b, \bar{c}_{p}\right)=q\left(\bar{c}_{q} ; b, \bar{c}_{q}\right)=q\left(b ; b, c_{q}\right)=I .
$$

From (23) it is seen that these numbers satisfy the inequalities

$$
c_{p}<\bar{c}_{p}<\bar{c}_{q}<c_{q}
$$

and (24) takes the form $c_{p} \leqq c \leqq c_{q}$. The three cases (28) are equivalent to the following:

Case $p . \quad c_{p} \leqq c \leqq \bar{c}_{p}$

Case $o . \quad \bar{c}_{p} \leqq c \leqq \bar{c}_{q}$.

Case $q . \quad \bar{c}_{q} \leqq c \leqq c_{q}$.

We now calculate $L_{c}(I ; b, c)$ in each of the three cases. In the first place, we have

$$
\begin{array}{ll}
p_{r}(r ; b, c)=\frac{-2}{(1+r)^{2}} \log \frac{b}{r}, & p_{c}(r ; b, c)=-\frac{1-c}{c(1+c)} \\
q_{r}(r ; b, c)=\frac{2}{(1-r)^{2}} \log \frac{b}{r}, & q_{c}(r ; b, c)=-\frac{1+c}{c(1-c)} .
\end{array}
$$

From these and the definition (30), we have

$$
L_{c}(I ; b, c)= \begin{cases}-\frac{1+c}{c(1-c)}-\left(\frac{1+r}{1-r}\right)^{2} \frac{1-c}{c(1+c)} \text { where } p(r ; b, c)=I & (\text { Case } p), \\ -\frac{2}{c I} \log \frac{b}{c} & \text { (Case } o), \\ -\frac{1-c}{c(1+c)}-\left(\frac{1-r}{1+r}\right)^{2} \frac{1+c}{c(1-c)} \text { where } q(r ; b, c)=I & (\text { Case } q) .\end{cases}
$$

In particular, the values of $L_{c}\left(I ; b, c_{p}\right)$ and $L_{c}\left(I ; b, c_{q}\right)$ are obtained from the first and last parts of (49) by putting $r=b$. Furthermore, we find from (49) that

$$
L_{c}\left(I ; b, \bar{c}_{p}\right)=-\frac{2\left(1+\bar{c}_{p}\right)}{\bar{c}_{p}\left(1-\bar{c}_{p}\right)}, \quad L_{c}\left(I ; b, \bar{c}_{q}\right)=-\frac{2\left(1-\bar{c}_{q}\right)}{\bar{c}_{q}\left(1+\bar{c}_{q}\right)}
$$

that is, both the left- and right-hand derivatives at $\bar{c}_{p}$ and $\bar{c}_{q}$ have these values. Thus $L_{c}(I ; b, c)$ exists everywhere and is continuous. 
It is evident from (49) that $L_{c}(I ; b, c)<0$ in all cases. Hence from (29) we see that the smallest $J$ for given $a$ and $b$ is obtained for $c=c_{q}$; we thus verify again that the hyperbolic expansion is maximized in this case. This does not however tell when $d$ itself is largest. For this purpose, we have to maximize $M(I ; b, c)$. From the definition (44) we find that

$$
M_{c}(I ; b, c)=-\frac{2 c}{1-c^{2}}-L_{c}(I ; b, c) .
$$

We must investigate the sign of $M_{c}(I ; b, c)$ in each of the cases $(47)$.

Case $p$. We see at once that $M_{c}(I ; b, c)>0$, so that the maximum value of $M(I ; b, c)$ does not occur in this interval.

Case $o$. We find from (50) that

$$
M_{c}\left(I ; b, \bar{c}_{p}\right)>0, \quad M_{c}\left(I ; b, \bar{c}_{q}\right)>,=,<0 \text { according as } \bar{c}_{q}<,=,>1 / 2 .
$$

Now $M_{c}(I ; b, c)$ is seen to be decreasing, so that it is negative, if at all, in a subinterval abutting $\bar{c}_{q}$. Hence $M(I ; b, c)$ is monotone increasing in the interval if $\bar{c}_{q} \leqq 1 / 2$, while if $\bar{c}_{q}>1 / 2$ it increases to a maximum and then decreases. In the latter case, the maximum is at a point $c>1 / 2$. For the condition $\bar{c}_{q}>1 / 2$ is equivalent to $q(1 / 2 ; b, 1 / 2)>I$ or $3 \log 2 b>I$; the condition $M_{c}(I ; b, 1 / 2)>0$ reduces to the same form if $c=1 / 2$ comes in Case $o$, and is trivial if it comes in Case $p$.

Case $q$. The condition $M_{c}(I ; b, c)>0$ is seen to reduce to

$$
(1+c)^{2}<(1+r)^{2} / 2 r \quad \text { where } q(r ; b, c)=I .
$$

Since $r$ increases with $c$, we see that (53) is more likely to be true the smaller $c$ is. Hence $M(I ; b, c)$ first increases and then decreases, or else is monotone increasing or decreasing. It starts to increase if $\bar{c}_{q}<1 / 2$, and increases throughout the interval if

$$
\left(1+c_{q}\right)^{2} \leqq(1+b)^{2} / 2 b .
$$

We note also that (53) is certainly true if $c \leqq 2^{1 / 2}-1$, since the right side is more than 2 ; and it is certainly false if $c \geqq 1 / 2$, since then $r \geqq c \geqq 1 / 2$, so that $(1+r)^{2} / 2 r \leqq 9 / 4 \leqq(1+c)^{2}$.

Putting together the results from the three cases, we see that either $M(I ; b, c)$ is monotone increasing in the whole interval $c_{p} \leqq c \leqq c_{q}$, or else it first increases and then decreases. Its largest value is at a point $c$ satisfying the following conditions: $c>\bar{c}_{p}$; either $\bar{c}_{q}<c<1 / 2, \bar{c}_{q}=c=1 / 2$, or $\bar{c}_{q}>c>1 / 2$; $c=c_{q}$ if and only if (54) is true; and if $c \neq c_{q}$ then $c>2^{1 / 2}-1$.

The conditions involved here may be expressed in terms of $a$ and $b$. In the first place,

$$
\bar{c}_{q}<,=,>1 / 2 \text { according as } a<,=,>1 / 8 b^{3} .
$$

Also, (54) with $c_{q}$ determined as the root of (39) is equivalent to 


$$
a \leqq \frac{c}{(1-c)^{2}}: \frac{b}{(1-b)^{2}} \quad \text { where }(1+c)^{2}=\frac{(1+b)^{2}}{2 b} .
$$

Substituting the value of $c$, this becomes

$$
a \leqq \frac{(1-b)^{2}\left[2 b\left(3-2 b+3 b^{2}\right)+(1+b)^{3}(2 b)^{1 / 2}\right]}{b\left(1-6 b+b^{2}\right)^{2}} .
$$

Using the form (56), we see that this condition is true whenever $b \leqq 1 / 2$, since then $c \geqq 1 / 2$; but that is is not true for $b>1 / 2$ and $a$ near 1 .

We now state the best upper bound for $d$ in the various cases. The cases are distinguished according to the point $c$ where $M(I ; b, c)$ is largest.

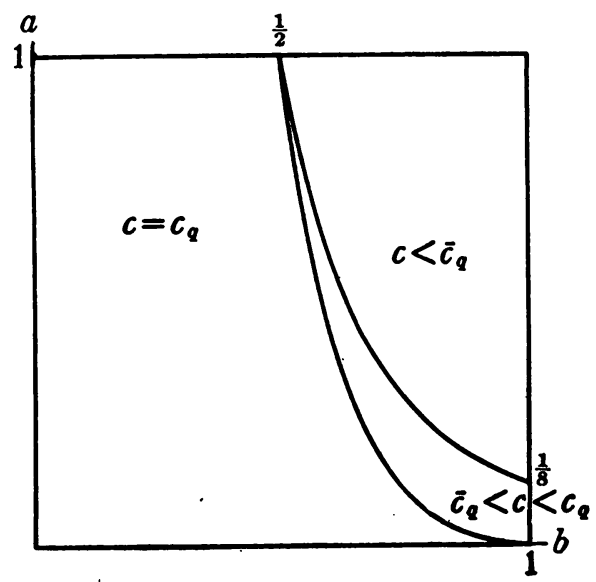

Fig. 1

Case 1. $c \leqq \bar{c}_{q}$. This is true if $\bar{c}_{q} \geqq 1 / 2$, or if $a \geqq 1 / 8 b^{3}$. The value of $c$ is found from $M_{c}(I ; b, c)=0$, using the formula of Case $o$. It is easily seen that the bound may be written in the form

(58) $\log d \leqq \log \frac{1-c^{2}}{1-b^{2}}-\frac{c^{2}}{1-c^{2}} \log \frac{b}{c} \quad$ where $\log \frac{b}{c}=\frac{c^{2}}{1-c^{2}} \log \frac{1}{a}$.

Case 2. $\bar{c}_{q}<c<c_{q}$. This is true if $a<1 / 8 b^{3}$ and (57) is false. Here

$$
\begin{aligned}
& \log d \leqq \log \frac{1-c^{2}}{1-b^{2}}-p(r ; b, c) \\
& \quad \text { where } q(r ; b, c)=\log \frac{1}{a} \text { and }(1+c)^{2}=\frac{(1+r)^{2}}{2 r} .
\end{aligned}
$$

Case 3. $c=c_{q}$. This holds if (57) is true, and in particular if $b \leqq 1 / 2$. We then have the result (38), which was previously obtained under more restrictive conditions. ure 1.

The values of $a$ and $b$ for which the various cases hold are shown in Fig- 
7. Relations between $a, c, d$. The lower bound for $d$ in terms of $a$ and $c$ is obtained from (42) by taking $b$ as large as possible, consistent with (31). If (40) is satisfied, then there is a largest value possible for $b$; the required lower bound for $d$ is (42) with $b$ determined from (37), which is seen to be the same as (36) with the same value of $b$ substituted. If (40) is false, then $b$ may be arbitrarily near to 1 , and (42) shows that there is no positive lower bound for $d$. Thus our previous results for this case are checked.

We now turn to the problem of finding the upper bound for $d$ in terms of $a$ and $c$. To do this, we have to eliminate $b$ from (43). This turns out to be the most difficult problem of all. In order to prevent this section from being unreasonably long, we shall omit a number of calculations; but some of these are quite similar to those given in $\$ 8$ for the case of unbounded functions.

Since $L(I ; b, c)$ is defined in (30) by different formulas according to which of the cases (28) holds, we must now consider in what intervals $b$ must lie in order that each of these cases may apply, when $I$ and $c$ are given. It is clear from (22) that all four functions

$$
p(b ; b, c), \quad p(c ; b, c), \quad q(c ; b, c), \quad q(b ; b, c)
$$

are increasing functions of $b$. As $b$ increases from $c$ to 1 , the four functions increase from 0 to certain limiting values.

$$
\begin{array}{ll}
p(1 ; 1, c)=\log \frac{(1+c)^{2}}{4 c}, & p(c ; 1, c)=\frac{1-c}{1+c} \log \frac{1}{c} \\
q(c ; 1, c)=\frac{1+c}{1-c} \log \frac{1}{c}, & q(1 ; 1, c)=+\infty
\end{array}
$$

We wish to determine values of $b_{p}, \bar{b}_{p}, \bar{b}_{q}, b_{q}$, not greater than 1 , which satisfy

$$
p\left(b_{p} ; b_{p}, c\right)=p\left(c ; b_{p}, c\right)=q\left(c ; b_{q}, c\right)=q\left(b_{q} ; b_{q}, c\right)=I,
$$

so far as this is possible. We can always find $b_{q}<1$; and we can find

$$
\begin{gathered}
b_{p} \leqq 1 \text { if } a \geqq 4 c /(1+c)^{2}, \quad \bar{b}_{p} \leqq 1 \text { if } a \geqq c^{(1-c) /(1+c)}, \\
b_{q} \leqq 1 \text { if } a \geqq c^{(1+c) /(1-c)} .
\end{gathered}
$$

The quantities $b_{p}, b_{p}, b_{q}, b_{q}$, so far as they exist, are seen from (23) to satisfy the inequalities

$$
b_{p}>\bar{b}_{p}>b_{q}>b_{q} .
$$

If any one of the quantities does not exist, we shall treat it in inequalities as if it were more than 1 . For example, $\bar{b}_{p}>1$ would mean that no $b_{p} \leqq 1$ can be found. If $b_{p} \geqq 1$, then $b \geqq b_{p}$ is impossible, since $b<1$ in any case; and $b<\bar{b}_{p}$ would impose no condition on $b$. With this interpretation, the three cases (28) take the form 


$$
\text { Case } p . \quad b_{p} \geqq b \geqq \bar{b}_{p} .
$$

Case $0 . \quad b_{p} \geqq b \geqq b_{q}$.

Case $q . \quad b_{q} \geqq b \geqq b_{q}$.

For some values of $a$ and $c$, not all the cases occur.

We now calculate $L_{b}(I ; b, c)$ in each of the three cases. Besides (48) we have

$$
p_{b}(r ; b, c)=\frac{1-r}{b(1+r)}, \quad q_{b}(r ; b, c)=\frac{1+r}{b(1-r)}
$$

From these we find that

$$
L_{b}(I ; b, c)= \begin{cases}\frac{2(1+r)}{b(1-r)} \text { where } p(r ; b, c)=I & (\text { Case } p), \\ \frac{2}{b I} \log \frac{b}{c} & \text { (Case } o), \\ \frac{2(1-r)}{b(1+r)} \text { where } q(r ; b, c)=I & (\text { Case } q) .\end{cases}
$$

We see that at $b_{p}$ and $b_{q}$ the derivative has the same value to the left and to the right.

It is clear that $L_{b}(I ; b, c)>0$ in all cases, so that $L(I ; b, c)$ is a monotone increasing function of $b$. Hence by (29), the smallest value of $J$ for given $a$ and $c$ is obtained by taking $b$ as small as possible. We thus verify that the hyperbolic expansion is maximized in this way.

We next consider the behavior of $L(I ; b, c)$ as $b \rightarrow 1$. In order for $b \rightarrow 1$ to be possible, we must have $b_{p} \geqq 1$. It may be shown that $L(I ; b, c)$ approaches a finite limit if $b_{p}>1$, and that $L(I ; b, c)+2 \log (1-b)$ approaches a finite limit if $b_{p}=1$. From this we find that

$$
\begin{array}{ll}
M(I ; b, c) \rightarrow+\infty & \text { as } b \rightarrow 1 \text { if } b_{p}>1, \\
M(I ; b, c) \rightarrow-\infty & \text { as } b \rightarrow 1 \text { if } b_{p}=1 .
\end{array}
$$

The first formula shows that no upper bound for $d$ in terms of $a$ and $c$ can be found if $b_{p}>1$. On the other hand, if $b_{p}=1$, then $d \rightarrow 0$ as $b \rightarrow 1$. In fact, if $d_{\text {min }}$ and $d_{\max }$ denote the smallest and largest values of $d$ for given $a, b, c$, it may be shown that if $a$ and $c$ have fixed values such that $b_{p}=1$, then

$$
d_{\max } / d_{\min } \rightarrow 4 / e
$$

We now turn to the consideration of

$$
M_{b}(I ; b, c)=\frac{2 b}{1-b^{2}}-L_{b}(I ; b, c) .
$$


From (66) we see that the condition $M_{b}(I ; b, c)<0$ at the points $b_{p}, b_{p}, b_{q}, b_{q}$ reduces to

$$
b_{p}<1, \quad b_{p}<((1+c) / 2)^{1 / 2}, \quad b_{q}<((1-c) / 2)^{1 / 2}, \quad b_{q}<1 / 2,
$$

respectively. Since $p\left(b_{p} ; b_{p}, G\right)=I$, the first condition is equivalent to $p(1 ; 1, c)>I$, and similarly for the others. The four conditions become

$$
\begin{aligned}
& a>4 c /(1+c)^{2}, \quad a>\left(2 c^{2} /(1+c)\right)^{(1-c) / 2(1+c)}, \\
& a>\left(2 c^{2} /(1-c)\right)^{(1+c) / 2(1-c)}, \quad a>c / 2(1-c)^{2} .
\end{aligned}
$$

These conditions are satisfied above the curves which are denoted by $p, \bar{p}, \bar{q}, q$, respectively, in Figure 2. We wish to know that the curves have the relative position shown in the figure. To verify that curve $\phi$ lies below curve $p$, we put

$$
\phi(c)=(3+5 c) \log 2+4 c \log c-(3+5 c) \log (1+c),
$$

and have to show $\phi(c)>0$ for $0<c<1$. Calculating the first and second derivatives, we find that $\phi^{\prime \prime}(c)>0$ and $\phi^{\prime}(1)=0$, hence $\phi^{\prime}(c)<0$ for $0<c<1$; then since $\phi(1)=0$, we find that $\phi(c)>0$ in the interval. By means of similar considerations, we can show that each other pair of curves intersects in exactly one point, and then by numerical calculation it is easily seen that the points of intersection lie as shown in the figure.

By a detailed study of the three cases (64), it may be shown that $M_{b}(I ; b, c)<0$, if at all, in a single subinterval of the whole interval $b_{p} \geqq b \geqq b_{q}$. It is clear then from (70) and (67) that a necessary and sufficient condition that $M(I ; b, c)$ should be monotone decreasing is that $b_{p} \leqq 1$ and $b_{q} \leqq 1 / 2$. It is seen that this is also the condition that the largest value of $M(I ; b, c)$ is for $b=b_{q}$.

It may be shown further that $M(I ; b, c)$ is decreasing in some subinterval for $(c, a)$ above the heavy broken curve in Figure 2. This curve is tangent to $\bar{q}$. The equation of the curve to the left of the point of tangency is

$$
a=\frac{c}{K(1-c)^{2}},
$$

where $K=2.31 \cdots$ is a constant. This part of the curve together with $q$ and $\bar{q}$ bound a region where $M(I ; b, c)$ is decreasing somewhere between $b_{q}$ and $\bar{b}_{q}$. The equation of the curve to the right of the point of tangency is

$$
c^{2}=\left[1+I-\left(2 I+I^{2}\right)^{1 / 2}\right] e^{I-\left(2 I+I^{2}\right) 1 / 2},
$$

where as usual $I=\log 1 / a$. This curve with $\bar{q}$ and $\bar{p}$ bounds a region where $M(I ; b, c)$ is decreasing somewhere between $\bar{b}_{q}$ and $\bar{b}_{p}$.

The three heavy curves in Figure 2 divide the unit square into 5 regions which are numbered from 1 to 5 . In these, $M(I ; b, c)$ has the following behaviour: 1 , decreasing throughout; 2 , increasing and then decreasing; 3 , de- 
creasing and then increasing; 4 , increasing, then decreasing, then increasing again; 5 , increasing throughout.

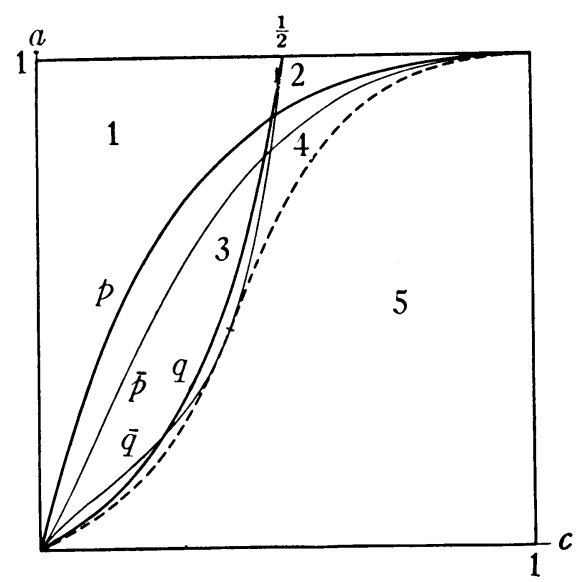

FIG. 2

We come finally to expressing the upper bound for $d$.in terms of $a$ and $c$. Only if $b_{p} \leqq 1$ is there any upper bound for $d$. In region 1 , for $b_{p} \leqq 1$ and $b_{q} \leqq 1 / 2$, the largest $d$ is attained for the smallest possible $b$, as was mentioned in (41). The result is then (38) with $b$ determined from (39). Region 2 is divided into two parts by $\bar{q}$. In the small region to the left we have

$$
\log d \leqq \log \frac{1-c^{2}}{1-b^{2}}-p\left(1-2 b^{2} ; b, c\right),
$$

where $b$ is the smaller root of $q\left(1-2 b^{2} ; b, c\right)=I$. To the right we have

$$
\log d \leqq \log \frac{1-c^{2}}{1-b^{2}}-\frac{b^{2}}{1-b^{2}} \log \frac{b}{c}
$$

where $b$ is the smaller root of $\log b / c=b^{2} I /\left(1-b^{2}\right)$.

Finally, we restate the most striking result: If values of $a$ and $c$ are given for which $b$ cannot have values arbitrarily near to 1 , then there is a maximum possible value for $d$; and the same is true for values of $a$ and $c$ which are limits of such values. But for all other values of $a$ and $c, d$ may have arbitrarily large values. The surprising part is that there is a sudden jump from one case to the other, rather than a gradual transition.

8. Appendix. As a supplement to our study of bounded univalent functions, we now consider univalent functions which are not supposed bounded. The results of this section are obtained from those of $\$ 5$ by a suitable passage to a limit; no use is made of $\$ \S 6$ and 7 .

Let $F(z)$ be a function which is regular and univalent for $|z|<1$, and for 
which $F(0)=0$ and $F^{\prime}(0)=1$. For any fixed $z_{0} \neq 0$ in the unit circle, we put

$$
b=\left|z_{0}\right|, \quad C=\left|F\left(z_{0}\right)\right|, \quad D=\left|F^{\prime}\left(z_{0}\right)\right| .
$$

We shall study the relations betweer $b, C, D$. Individually, the quantities satisfy only the inequalities

$$
0<b<1, \quad 0<C, \quad 0<D .
$$

Now any such $F(z)$ can be approximated by bounded functions of the same type, if the bound is allowed to vary. Hence any possible values of $b, C, D$ can be approximated for bounded functions. Conversely, on the basis of [5], the univalent functions form a normal family, so that any values of $b, C, D$ with $0<b<1$ which can be approximated can also be assumed. Hence the values possible for $b, C, D$ are those attained for bounded $F(z)$ together with the limit points satisfying $0<b<1$.

If $F(z)$ is bounded, we can choose $a>0$ so that $|a F(z)|<1$ for $|z|<1$. We then put

$$
f(z)=a F(z),
$$

so that $f(z)$ is a function of the class previously considered, and $f^{\prime}(0)=a$. Thus $a$ and $b$ have the same meaning as before, and

$$
c=a C, \quad d=a D .
$$

From the known relations between $a, b, c, d$, we obtain the relations between $a, b, C, D$; by eliminating $a$, we find the relations between $b, C, D$.

Relations between $b$ and $C$. If in formula (31) we put $c=a C$, and then let $a \rightarrow 0$, we obtain the well known inequalities $\left({ }^{6}\right)$

$$
\frac{b}{(1+b)^{2}} \leqq C \leqq \frac{b}{(1-b)^{2}}
$$

The bounds are attained for the function

$$
F(z)=\frac{z}{(1-z)^{2}}
$$

for $z_{0}<0$ and $z_{0}>0$, respectively. The function [6] maps $|z|<1$ onto the $w$-plane excluding those points for which $w \leqq-1 / 4$.

Relations between $b, C, D$. We remark first that the required inequalities are not obtained from the relations between $b, c, d$ by passing to a limit. If in (34) we put $c=a C$ and $d=a D$, and let $a \rightarrow 0$, we obtain Nevanlinna's result $\left({ }^{7}\right)$

(6) See for example L. Bieberbach, Lehrbuch der Funktionentheorie, vol. 2, chap. 1, \$9.

(7) R. Nevanlinna, Über die konforme Abbildungen von Sterngebieten, Finska VetenskapsSocieteten Förhandlingar, vol. 63 (1921), no. 6, p. 18. 


$$
\frac{C(1-b)}{b(1+b)} \leqq D \leqq \frac{C(1+b)}{b(1-b)} .
$$

This inequality gives the best bounds for $D / C$ in terms of $b$. It does not however give the best bounds for $D$ in terms of $b$ and $C$, since either of the equalities in (34) is attained only for a certain positive value of $a$.

The sharp lower bound for $D$ in terms of $b$ and $C$ is easily obtained. In (42) we put $c=a C, d=a D$, and let $a \rightarrow 0$, which gives

$$
D \geqq \frac{1-b^{2}}{b^{2}} C^{2} \text {. }
$$

It may be verified that the equality is attained for a function mapping $|z|<1$ on the $w$-plane with slits along the positive and negative real axes. For a given $b$, the slits may be so chosen that $C$ has any possible value. The equality is attained for any positive or negative $z_{0}$.

We turn now to the problem of finding the upper bound for $D$ in terms of $b$ and $C$. We start by introducing the functions

[9]

$$
P(r ; b, C)=\log \frac{r}{C(1+r)^{2}}+\frac{1-r}{1+r} \log \frac{b}{r},
$$

$$
Q(r ; b, C)=\log \frac{r}{C(1-r)^{2}}+\frac{1+r}{1-r} \log \frac{b}{r},
$$

for $0<r \leqq b$; for $r=0$ we put

$$
P(0 ; b, C)=Q(0 ; b, C)=\log b / C,
$$

which is the limiting form of [9]. The functions (21) are related to these by the equations

$$
\begin{aligned}
& p(r ; b, c)=p(r ; b, a C)=P(r ; b, C)-\log \frac{a}{(1+a C)^{2}} \\
& q(r ; b, c)=q(r ; b, a C)=Q(r ; b, C)-\log \frac{a}{(1-a C)^{2}}
\end{aligned}
$$

The function $P(r ; b, C)$ is a decreasing and $Q(r ; b, C)$ an increasing function of $r$; both increase with $b$, and decrease as $C$ increases. The partial derivatives have the values

$$
\begin{aligned}
& P_{r}(r ; b, C)=\frac{-2}{(1+r)^{2}} \log \frac{b}{r}, P_{b}(r ; b, C)=\frac{1-r}{b(1+r)}, P_{C}(r ; b, C)=-\frac{1}{C} . \\
& Q_{r}(r ; b, C)=\frac{2}{(1-r)^{2}} \log \frac{b}{r}, Q_{b}(r ; b, C)=\frac{1+r}{b(1-r)}, Q_{C}(r ; b, C)=-\frac{1}{C} .
\end{aligned}
$$


The three cases (28) take the following limiting forms as $a \rightarrow 0$ :

$$
\begin{gathered}
P(b ; b, C) \leqq 0 \leqq P(0 ; b, C), \quad P(0 ; b, C) \leqq 0 \leqq Q(0 ; b, C), \\
Q(0 ; b, C) \leqq 0 \leqq Q(b ; b, C) .
\end{gathered}
$$

These are equivalent to

$$
C \leqq b, \quad C=b, \quad C \geqq b,
$$

respectively, where $C$ of course satisfies [5].

In Cases $p$ and $q$, we determined $r$ from $p(r ; b, c)=I$ and $q(r ; b, c)=I$, respectively. These equations are the same as

$$
P(r ; b, C)+2 \log (1+a C)=0, \quad Q(r ; b, C)+2 \log (1-a C)=0 .
$$

As $a \rightarrow 0$, their roots approach those of

$$
P(r ; b, C)=0, \quad Q(r ; b, C)=0 .
$$

Putting $c=a C$ and $d=a D$ in (43), and letting $a \rightarrow 0$, we find that

$$
\log D \leqq M(b, C)
$$

where

$$
M(b, C)=\log \frac{1}{1-b^{2}}-L(b, C),
$$

$L(b, C)$ being defined for all possible values of $b$ and $C$ by

[19] $L(b, C)=\left\{\begin{array}{l}Q(r ; b, C) \text { where } r \text { satisfies } P(r ; b, C)=0, \text { if } C \leqq b, \\ P(r ; b, C) \text { where } r \text { satisfies } Q(r ; b, C)=0, \text { if } C \geqq b .\end{array}\right.$

These two formulas correspond to Cases $p$ and $q$ in (30). For the case $C=b$, either of these, and also Case $o$, leads to the result

$$
D \leqq \frac{1}{1-b^{2}}
$$

if $C=b$.

It may be verified that the equality in [20] is attained for a function $F(z)$ which maps $|z|<1$ on the $w$-plane slit to infinity at one or both ends of the perpendicular bisector of the segment joining 0 and $f\left(z_{0}\right)$. We see from [8] and [17] that when $C$ has its smallest value, we must have $D=(1-b) /(1+b)^{3}$, and that when $C$ has its largest value, $D=(1+b) /(1-b)^{3}$; in these cases the equalities in both [8] and [17] are attained for the function [6]. We know that in any case, there is some function for which the equality in [17] is attained; but the extremal function does not seem to be of a very simple sort except in the three cases mentioned.

Relations between $b$ and $D$. From [8] we see that $D$ has its smallest value when $C$ has its smallest value, determined from [5]. This gives 


$$
D \geqq \frac{1-b}{(1+b)^{3}} .
$$

The equality is attained for the function [6] with $z_{0}<0$.

We next determine the upper bound for $D$. In the first part of [19], as $C$ increases, $r$ decreases, and hence $Q(r ; b, C)$ decreases; in the second part, as $C$ increases, $r$ increases, and hence $P(r ; b, C)$ decreases. Hence in either case, $L(b, C)$ is decreasing, or $M(b, C)$ is increasing. Thus the largest value of $D$ is obtained when $C$ has its largest value, and hence

$$
D \leqq \frac{1+b}{(1-b)^{3}} .
$$

The equality is attained for the function [6] with $z_{0}>0$.

We may also verify that $M(b, C)$ is increasing by calculating its partial derivative. Making use of [12], we find that

$$
M_{C}(b, C)=\left\{\begin{array}{l}
\frac{1}{C}\left[\left(\frac{1+r}{1-r}\right)^{2}+1\right] \text { where } P(r ; b, C)=0, \text { if } C \leqq b, \\
\frac{1}{C}\left[\left(\frac{1-r}{1+r}\right)^{2}+1\right] \text { where } Q(r ; b, C)=0, \text { if } C \geqq b,
\end{array}\right.
$$

so that $M_{C}(b, C)>0$ throughout.

The inequalities [21] and [22] ("distortion theorem") are well known, and were used to derive [5] in the original approach to this subject $\left.{ }^{8}\right)$.

Remarks about $D / C$ and $D / C^{2}$. From [8] we see that $D / C$ has its smallest value when $C$ is smallest. From [23] we see that $M(b, C)-\log C$ is an increasing function, and hence $D / C$ has its largest value when $C$ is largest. Thus we are again led to [7].

On the other hand, [8] shows that $D / C^{2}$ can reach its smallest value for any $C$. From [23] we see that $M(b, C)-2 \log C$ has its maximum for $C=b$, so that $D / C^{2}$ attains its largest value only in this case. We obtain the inequalities

$$
\frac{1-b^{2}}{b^{2}} \leqq \frac{D}{C^{2}} \leqq \frac{1}{b^{2}\left(1-b^{2}\right)} .
$$

This result gives the bounds for the derivative of $1 / F(z)$, or for the derivative of a function univalent in the exterior of the unit circle and leaving $\infty$ fixed. The problem was solved in this form by Löwner $\left({ }^{9}\right)$ (without using the "method of Löwner").

(8) See Bieberbach, loc. cit.

() K. Löwner, Über Extremumsätze bei der konformen Abbildungen des Äusseren des Einheitskreises, Mathematische Zeitschrift, vol. 3 (1919), pp. 65-77. 
Relations between $C$ and $D$. To find the lower bound for $D$ in terms of $C$, we must eliminate $b$ from [8]. Evidently the larger $b$ is, the smaller $D$ may be. Now $b$ is restricted only by [5]. If $C \geqq 1 / 4, b$ may have values arbitrarily near to 1 , and hence there is no positive lower bound for $D$. But if $C<1 / 4$, then the lower bound for $D$ is given by [8] with $b$ determined from $b /(1+b)^{2}$ $=C$. Solving for $b$ and substituting gives

$$
2 D \geqq 1-4 C+(1-2 C)(1-4 C)^{1 / 2} \quad \text { for } C<1 / 4 .
$$

The equality is attained for the function [6] with $z_{0}<0$.

We now turn to the problem of finding the upper bound for $D$ in terms of $C$. We first consider the behavior of $L(b, C)$ as $b \rightarrow 1$. In order that $b \rightarrow 1$ should be possible, we must have $C \geqq 1 / 4$. If $C \geqq 1$, then the second part of [19] applies. As $b \rightarrow 1, r$ decreases, and $L(b, C)$ increases to a finite limit. If $C<1$, then the first part of [19] applies for $b$ near 1 . As $b \rightarrow 1, r$ increases, and $L(b, C)$ increases; and $L(b, C)$ approaches a finite limit unless $r \rightarrow 1$. Now if $r \rightarrow 1$ as $b \rightarrow 1$, we must have $P(1 ; 1, C)=0$, or $C=1 / 4$. Hence if $C>1 / 4, L(b, C)$ increases to a finite limit as $b \rightarrow 1$. The case $C=1 / 4$ remains to be considered. Here $r$ is determined from $P(r ; b, 1 / 4)=0$, which is equivalent to

$$
\log b=\log r+\frac{1+r}{1-r} \log \frac{(1+r)^{2}}{4 r} .
$$

From this we find that

$$
1-r \sim 2(1-b)
$$
as $b \rightarrow 1$.

Using this in the formula $L(b, 1 / 4)=Q(r ; b, 1 / 4)$, we find that

$$
L(b, 1 / 4)+2 \log (1-b) \rightarrow 1
$$

as $b \rightarrow 1$.

From these results we find that

$$
\begin{array}{ll}
M(b, C) \rightarrow+\infty & \text { as } b \rightarrow 1 \text { if } C>1 / 4, \\
M(b, C) \rightarrow-\infty & \text { as } b \rightarrow 1 \text { if } C=1 / 4 .
\end{array}
$$

The first formula shows that no upper bound for $D$ can be found if $C>1 / 4$. The second part may be written more accurately as

$$
M(b, 1 / 4)-\log (1-b) \rightarrow-1-\log 2 \quad \text { as } b \rightarrow 1 .
$$

If we denote by $D_{\min }$ and $D_{\max }$ the smallest and largest values of $D$ which are possible for given $b$ and $C$, then for $C=1 / 4$ we have from [8] and [30]

$$
D_{\min } \sim \frac{1-b}{8}, \quad D_{\max } \sim \frac{1-b}{2 e}, \quad \frac{D_{\max }}{D_{\min }} \rightarrow \frac{4}{e} \quad \text { as } b \rightarrow 1 .
$$

We now consider the derivative of $M(b, C)$. Using [12], we find that 


$$
M_{b}(b, C)=\left\{\begin{array}{l}
\frac{2 b}{1-b^{2}}-\frac{2(1-r)}{b(1+r)} \text { where } Q(r ; b, C)=0, \text { if } b \leqq C, \\
\frac{2 b}{1-b^{2}}-\frac{2(1+r)}{b(1-r)} \text { where } P(r ; b, C)=0, \text { if } b \geqq C .
\end{array}\right.
$$

Hence at $b=C, M_{b}(b, C)$ has the same value to the left and to the right.

A remark which will be useful below is the following. For any fixed $b$ the root $r$ of $P(r ; b, C)=0$ decreases from $b$ to 0 as $C$ increases from its smallest possible value to $b$; and the root $r$ of $Q(r ; b, C)=0$ increases from 0 to $b$ as $C$ increases from $b$ to its largest possible value. Hence if $0 \leqq r \leqq b$, the equation $P(r ; b, C)=0$ determines a value of $C \leqq b$, and $Q(r ; b, C)$ determines a $C \geqq b$, both satisfying [5].

Now the equation

$$
M_{b}(b, C)=0
$$

requires in the first case that $r=1-2 b^{2}$ and in the second that $r=2 b^{2}-1$. Since we must have $0 \leqq r \leqq b, M_{b}(b, C)$ vanishes only in the following cases:

$$
M_{b}(b, C)=0\left\{\begin{array}{l}
\text { if } 1 / 2 \leqq b \leqq 2^{-1 / 2} \text { and } Q\left(1-2 b^{2} ; b, C\right)=0 \\
\text { if } 2^{-1 / 2} \leqq b<1 \text { and } P\left(2 b^{2}-1 ; b, C\right)=0
\end{array}\right.
$$

These may be combined in the statement that $M_{b}(b, C)=0$ only along the curve

$$
4 C=b^{-5+1 / b^{2}}\left|1-2 b^{2}\right|^{2-1 / b^{2}}, \quad 1 / 2 \leqq b<1,
$$

which from the preceding paragraph must lie between the bounds [5]. To study this curve, we put $h=1 / b^{2}$ and

Then

$$
\phi(h)=2 \log 4 C .
$$

$$
\phi(h)=(h+1) \log h-2(h-2) \log |h-2|,
$$

where for $h=2$ we interpret the right side as its limiting value $3 \log 2$. Differentiating, we have

$$
\phi^{\prime}(h)=\log h-2 \log |h-2|-1+\frac{1}{h}, \quad \phi^{\prime \prime}(h)=\frac{1}{h}-\frac{2}{h-2}-\frac{1}{h^{2}} .
$$

We see that $\phi^{\prime \prime}(h)>0$ for $1<h<2$, and $\phi^{\prime \prime}(h)<0$ for $2<h .<4$. Using this we find that $\phi^{\prime}(h)$ increases from 0 to $+\infty$ as $h$ increases from 1 to 2 , and then decreases to $-3 / 4$ as $h$ increases to 4 . We must have $\phi^{\prime}(h)=0$ at some point between 2 and 4 , and in fact for $h=3.27 \ldots$; and here $\phi(h)$ has its maximum. Therefore, in [34], $C$ increases from 2 when $b=1 / 2$ to a maximum $K=2.31 \cdots$ for $b=0.55 \cdots$, and then decreases to $1 / 4$ as $b$ increases to 1 . Figure 3 shows 
[34] as a broken curve and the bounds [5] as solid curves.

The curve [34] divides the region defined by [5] into two parts. There is no difficulty in seeing that $M_{b}(b, C)<0$ in the lower part, and $M_{b}(b, C)>0$ in the upper part. Hence $M(b, C)$ is a decreasing function of $b$ if $C \leqq 1 / 4$; if $1 / 4<C \leqq 2$, it first decreases, and then increases to $+\infty$; if $2<C<K$, it first

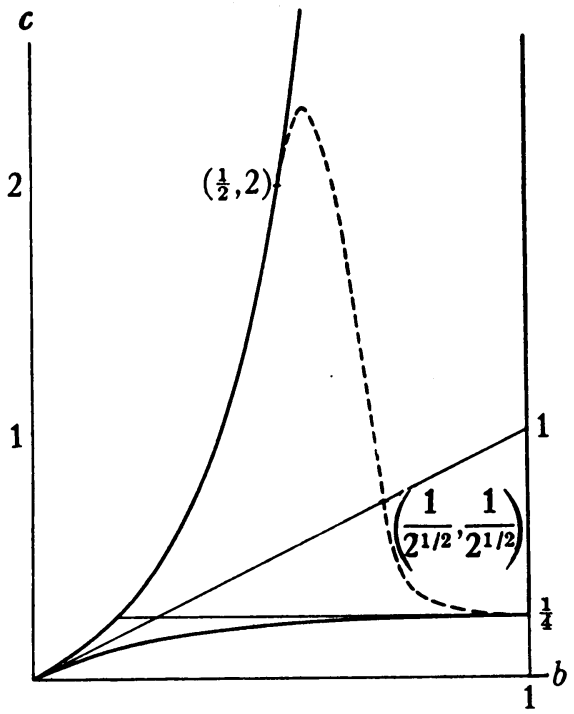

Fig. 3

increases, then decreases, then increases to $+\infty$; and if $C \geqq K, M(b, C)$ increases throughout, and approaches $+\infty$ as $b \rightarrow 1$. There is an upper bound for $D$ only if $C \leqq 1 / 4$, and then it is attained when $b$ has its smallest possible value. Hence the bound is given by [22] with $b$ determined from $b /(1-b)^{2}=C$. Substituting this value of $b$, we find that

$$
2 D \leqq 1+4 C+(1+2 C)(1+4 C)^{1 / 2} \quad \text { for } C \leqq 1 / 4 \text {. }
$$

The equality is attained for the function [6] with $z_{0}>0$. It is to be noted that the upper bound for $D$ in terms of $C$ increases from 1 to $2.06 \cdots$ as $C$ increases from 0 to $1 / 4$, and then jumps to $+\infty$.

University of California, Berkeley, Calif. 